\title{
Correlated Motion of Electrons on the Au(111) Surface: Anomalous Acoustic Surface-Plasmon Dispersion and Single-Particle Excitations
}

\author{
L. Vattuone, ${ }^{1}$ M. Smerieri, ${ }^{1}$ T. Langer, ${ }^{2}$ C. Tegenkamp, ${ }^{2}$ H. Pfnür, ${ }^{2}$ V. M. Silkin, ${ }^{3,4,5}$ E. V. Chulkov, ${ }^{3,4,6}$ \\ P. M. Echenique, ${ }^{3,4,6}$ and M. Rocca ${ }^{1}$ \\ ${ }^{1}$ Dipartimento di Fisica dell'Universitá di Genova and IMEM-CNR Unitá Operativa di Genova, \\ Via Dodecaneso 33, 15146 Genova, Italy \\ ${ }^{2}$ Institut für Festkörperphysik, Abteilung Atomare und Molekulare Strukturen, Leibniz Universität Hannover, \\ Appelstraße 2, D-30167 Hannover, Germany \\ ${ }^{3}$ Departamento de Física de Materiales, Facultad de Ciencias Químicas, Universidad del País Vasco, \\ Apartado 1072, 20080 San Sebastián/Donostia, Spain \\ ${ }^{4}$ Donostia International Physics Center (DIPC), Paseo de Manuel Lardizabal 4, 20018 San Sebastián/Donostia, Spain \\ ${ }^{5}$ IKERBASQUE, Basque Foundation for Science, 48011 Bilbao, Spain \\ ${ }^{6}$ Centro de Física de Materiales CFM-Materials Physics Center MPC, Centro Mixto CSIC-UPV/EHU, \\ Paseo de Manuel Lardizabal 5, 20018 San Sebastián/Donostia, Spain
}

(Received 8 October 2012; published 22 March 2013)

\begin{abstract}
The linear dispersion of the low-dimensional acoustic surface plasmon (ASP) opens perspectives in energy conversion, transport, and confinement far below optical frequencies. Although the ASP exists in a wide class of materials, ranging from metal surfaces and ultrathin films to graphene and topological insulators, its properties are still largely unexplored. Taking $\mathrm{Au}(111)$ as a model system, our combined experimental and theoretical study revealed an intriguing interplay between collective and single particle excitations, causing the ASP associated with the Shockley surface state to be embedded within the intraband transitions without losing its sharp character and linear dispersion.
\end{abstract}

DOI: 10.1103/PhysRevLett.110.127405

PACS numbers: 78.68.+m, 71.45.Gm, 79.20.Uv

One of the most attractive aspects of plasmonics-an explosively growing interdisciplinary research field-is the ability to squeeze light into subwavelength regions thanks to the properties of surface plasmon polaritons (SPP) [1,2], an excitation resulting from the interaction of photons with surface plasmons [3]. In particular, light confinement (i.e., the ratio between the wavelength of the electromagnetic radiation and of the induced plasmonic excitation) in the plane parallel to the surface takes place because of the several times lower velocity of the SPP with respect to light, implying that at any given excitation frequency, the wavelength of the SPP is shortened by the same ratio.

Even higher in-plane confinements can be achieved with plasmons associated with a two dimensional electronic gas, notably in graphene [4], since their dispersion is much flatter than that of SPP. The 2D plasmon energy $\omega_{2 \mathrm{DP}}$ varies as $\sim \sqrt{q_{\|}}$for small in-plane momentum $q_{\|}$ with $\omega_{2 \mathrm{DP}} \rightarrow 0$ as $q_{\|} \rightarrow 0$ [5], which allows for extraordinary light confinement from subterahertz to midinfrared frequencies by use of appropriate nanostructures providing the necessary momentum transfer.

Recently, an in-plane confinement rate as high as 40 was attained in graphene sheets grown on a dielectric substrate $[6,7]$. An even higher value of $\sim 100$ was achieved for graphene nanostructures [8], making use of the 2D plasmon supported by the 2D electron gas of doped graphene. However, the $\sim \sqrt{q_{\|}}$dispersion makes a distortionless propagation of nonmonochromatic signals inherently impossible, since the different frequencies components propagate at different velocities. This drawback can be overcome using a plasmon energy with a linear rather than a square root dispersion. The former is expected whenever a partially filled electronic 2D band is coupled and shielded by other 2D or 3D electron gases [9], and is thus a fairly general concept. This is, in particular, the case for metal surfaces supporting an electronic Shockley surface state (SS) with band dispersion crossing the Fermi level [10].

Such a mode, called an acoustic surface plasmon (ASP), was observed, in addition to the conventional surface plasmons, for a variety of noble and simple metal surfaces [11-15]. Recently, a similar mode was reported also for graphene grown on a metallic substrate [16,17], providing the 2D and the 3D electron gases, respectively.

The origin of the ASP is traced to the incomplete screening of the Coulomb interaction due to the mutual motion of carriers in energy bands with higher (bulk electron system) and lower (SS) Fermi velocity [18], resulting in the dispersion relation

$$
\omega_{\mathrm{AP}}=\alpha \cdot v_{\mathrm{F}}^{\mathrm{SS}} \cdot q_{\|},
$$

where $v_{\mathrm{F}}^{\mathrm{SS}}$ is the SS Fermi velocity and the coefficient $\alpha$ is only slightly larger than unity [18]. Since $v_{\mathrm{F}}^{\mathrm{SS}}$ is sensitive to surface chemistry, the extreme confinement mentioned above can be tailored over a wide range of frequencies, 
which differs significantly from the energy region of operation in conventional plasmonics. Last but not least, the typical energy of the ASP (from zero up to $\approx 1 \mathrm{eV}$ ) makes it a, so far neglected, channel of dissipation for the excess energy released in surface reactions.

Despite these promising perspectives, the physics underlying the ASP is still not fully understood, giving rise to unexpected properties, as we shall show below. For $\mathrm{Be}(0001)$ [11] and $\mathrm{Cu}(111)$ [13], $\alpha$ came out to be in accord with theoretical forecast, but for $\mathrm{Au}$-an important plasmonic material-an ASP dispersion significantly larger than expected was recently observed by high resolution electron energy loss spectroscopy (HREELS) [12]. In this experiment, EEL signals up $2 \mathrm{eV}$ were indeed reported, far exceeding the maximum value predicted by theory, thus questioning our present understanding of low-energy screening at surfaces.

In order to solve this intriguing issue, we reinvestigated the electronic excitation spectrum on $\mathrm{Au}(111)$ by HREELS and energy loss spectroscopy-low energy electron diffraction (ELS-LEED) [19], combined with extended ab initio time dependent density functional theory calculations.

The essential physics of the ASP is captured by the following model. Firstly, let us consider a conventional one-component $2 \mathrm{D}$ electron gas with the band structure sketched in Fig. 1(a), dielectric function $\epsilon$ and loss function $\operatorname{Im} 1 / \epsilon$ reported at a given momentum transfer $q_{\|}$in Fig. 1(b). The latter presents only a broad unstructured background caused by the excitation of incoherent intraband electron-hole pairs, while the conventional 2D plasmon [5] is located at much higher energy [20]. Although a zero-crossing of $\operatorname{Re} \epsilon$ (marked by the light red arrow) occurs in this energy region, it does not give rise to a peak in the loss function since it coincides with a maximum in $\operatorname{Im} \epsilon$ and the corresponding mode is heavily damped due to decay into incoherent electron-hole pair excitations. Secondly, let us consider a two-component electron gas, characterized by different Fermi velocities $v_{\mathrm{F}}^{1}$ and $v_{\mathrm{F}}^{2}$ and a band structure like the one shown in Fig. 1(c). At any small $q_{\|}$, the corresponding $\epsilon$, shown in Fig. 1(d), presents two peaks in $\operatorname{Im} \epsilon$ due to the intraband transitions within each band, while the real part crosses zero three times. The crossings marked by light red arrows are similar to that in Fig. 1(b) and are silent in the loss function. The zero marked by the thick red arrow corresponds, on the other hand, to a well-defined peak in the loss function since it is associated with a local minimum in $\operatorname{Im} \epsilon$. Since it lies just above the edge of single particle transitions within the band with the lower Fermi velocity, it corresponds to a collective mode screened by the electrons located in the other band. At small momenta, the energies of both peaks in $\operatorname{Im} \epsilon$ disperse linearly with momentum [20], and vanish for $q_{\|} \rightarrow 0$. Therefore, the peak in the loss function trapped between them does the same and its dispersion can be described by Eq. (1), with $v_{\mathrm{F}}^{1}$ as a reference.
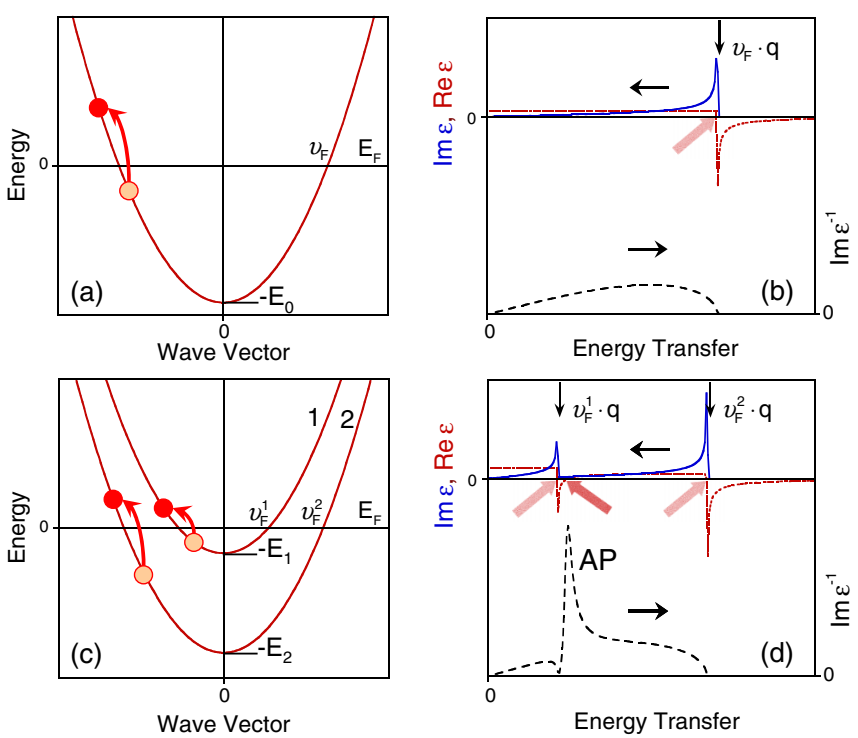

FIG. 1 (color online). Schematic of electronic excitations and decay in single- (a,b) and double-component (c,d) 2D electron gases: Energy dispersion as a function of wave vector (panels a and c) with single electron intraband transitions (red arrows). In (b) and (d), the dielectric function $\epsilon$ with real (dotted red lines) and imaginary parts (solid blue lines), and the loss function $\operatorname{Im} \epsilon^{-1}$ (dashed black lines) are plotted for an arbitrary, but given small momentum transfer. Unobservable low-energy resonances $(\operatorname{Re} \epsilon=0)$ located at $\omega=v_{\mathrm{F}} \cdot q_{\|}$coinciding with a peak in $\operatorname{Im} \epsilon$ (vertical arrow) are marked by thick light red arrows.

This basic picture can be directly applied to dilute electronic systems like semiconducting superlattices with a spatial separation of the components [21]. Although, in the case of a metal surface, other important issues must be taken into account explicitly (such as the replacement of one of the components by the bulk electron bands, the spatial superposition of surface and bulk states, and the numerous interband transitions), the above schematic picture still captures the essential physics [18].

The $\mathrm{Au}(111)$ single crystal is cleaned by sputtering and annealing cycles until a well-ordered low energy electron diffraction pattern was obtained. An electron beam of energy between 6 and $67 \mathrm{eV}$, monochromatized by a cylindrical deflector analyzer to an energy resolution $\approx 10 \mathrm{meV}$ (full width at half maximum), impinges onto the surface at a well-defined angle of incidence and along one of the high symmetry directions $\overline{\Gamma K}$ and $\overline{\Gamma M}$ (see inset of Fig. 2). Electrons scattered in plane at well-defined angles are analyzed in energy by an electrostatic deflector. The electron energy loss and the angle of scattering allow us to determine the exchanged momentum parallel to the surface plane $q_{\|}$of the excitation being created from energy and momentum conservation. $q_{\|}$is, thereby, calibrated with respect to the position in reciprocal space of the diffraction spots of the herringbone surface reconstruction of $\mathrm{Au}(111)$. Further experimental details as well as a 


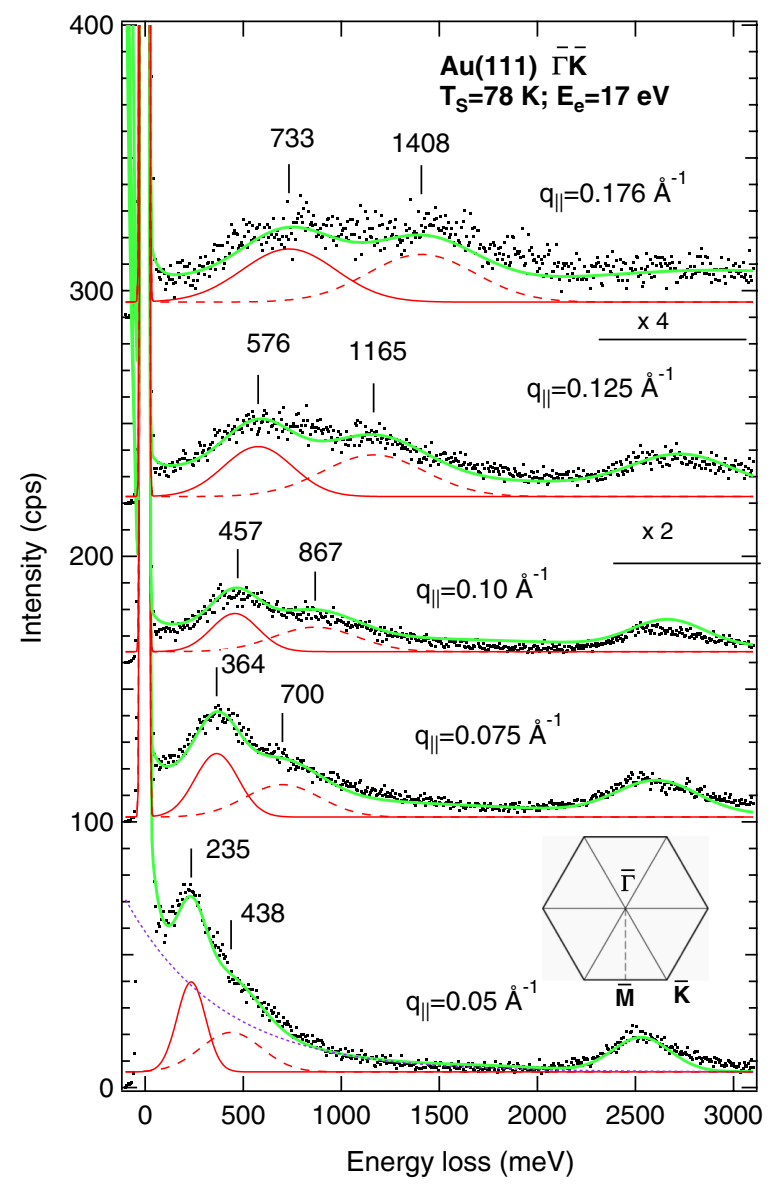

FIG. 2 (color online). Energy loss spectroscopy-low energy electron diffraction spectra recorded for different $q_{\|}$. Thick green lines are best fits to the data. Two peaks can clearly be identified in the loss spectra (red continuous and dashed lines, respectively). The contribution of the Drude tail (dotted) is shown only for the lowest spectrum for sake of clarity. The inset shows the 2D Brillouin zone of $\mathrm{Au}(111)$ with $\overline{\Gamma K}$ and $\overline{\Gamma M}$ symmetry directions.

description of the surface reconstruction are reported in the Supplemental Material [22].

In Fig. 2, we report a typical series of spectra for $q_{\|}$along $\overline{\Gamma K}$. In addition to the ordinary surface plasmon at $2.5 \mathrm{eV}$, showing a very weak dispersion [22], we observe a further loss at lower energy, which shifts to higher values with $q_{\|}$. It has an asymmetric line shape with a high energy tail evolving into a well-separated peak above $q_{\|}=0.1 \AA^{-1}$. For quantitative fits, the loss was described by two Gaussians plus a background consisting of an exponential (Drude tail [23]) and a constant. The energy positions of both peaks, their full width at half maximum (FWHM) and their absolute intensities resulting from Gaussian fits are plotted in Fig. 3 vs $q_{\|}$. The FWHMs were corrected for the broadening caused by the finite acceptance angle of the instrument.

From this figure, it is apparent that: (a) loss I exhibits a linear dispersion with the same slope within error along both azimuths. At $78 \mathrm{~K}$, the linear best fit $E_{I}=a+b q_{\|}$
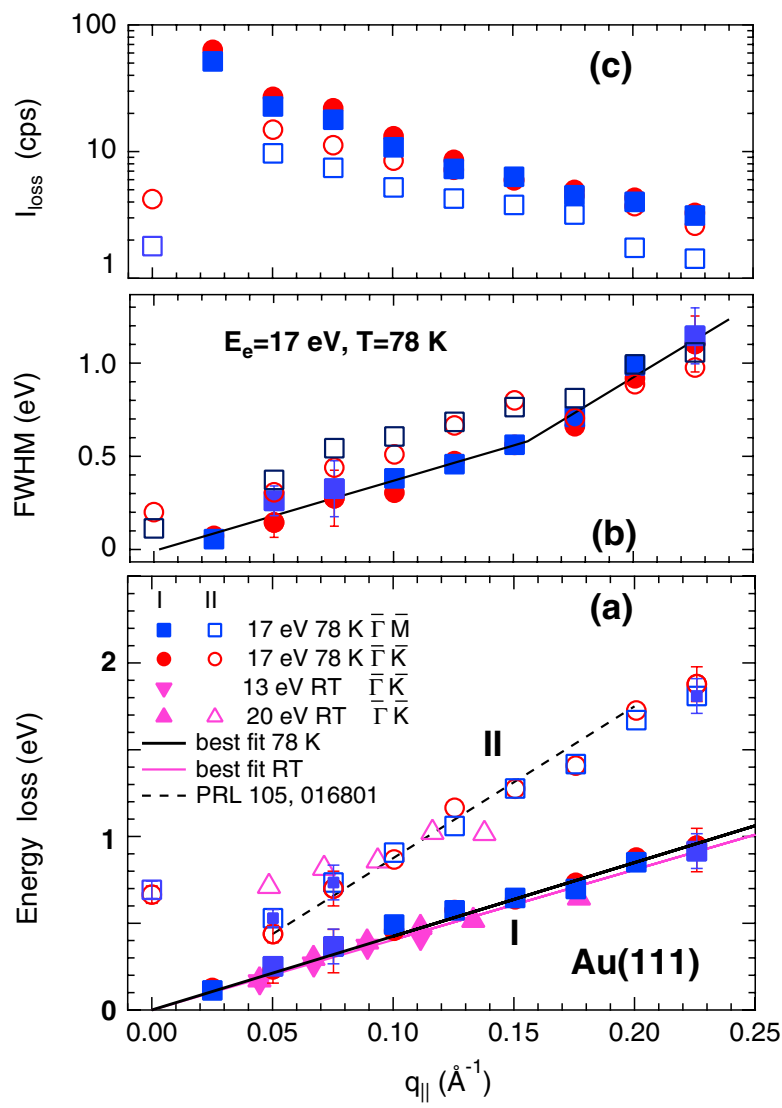

FIG. 3 (color online). (a): Energy losses I and II as a function of $q_{\|}$at $78 \mathrm{~K}$ and at room temperature RT obtained by fitting the experimental data for momentum transfers $q \|$ directed along $\overline{\Gamma K}$ (red) and $\overline{\Gamma M}$ (blue). Full symbols correspond to loss I, empty symbols to loss II in all panels. The lines represent the linear best fits to the corresponding data set. The error bars are obtained by using standard routines [38]. (b): FWHM vs $q_{\|}$for peaks I and II. (c): Intensity of the two losses $I_{\text {loss }}$ at constant impinging current.

yields $a=(0.03 \pm 0.03) \mathrm{eV}$ and $b=(3.95 \pm 0.26) \mathrm{eV} \cdot \AA$. Forcing $a=0$, we obtain a slope of $(4.25 \pm 0.14) \mathrm{eV} \cdot \AA$ (black line in Fig. 3). Since $v_{F}=7.65 \times 10^{5} \mathrm{~m} / \mathrm{s}$ for $\mathrm{Au}(111)[24,25]$, this mode is compatible with an ASP with $\alpha \approx 0.8$. The RT data are systematically lower than those obtained at $78 \mathrm{~K}$, where the fit forcing $a=0$ yields a slope of $(4.04 \pm 0.09) \mathrm{eV} \cdot \AA$. The difference, albeit at the limit of experimental error, is consistent with the destabilization of the Shockley SS with increasing $T$ $[24,26]$. (b) Comparing the present measurements with those of Ref. [12] (dashed line), it is evident that loss II coincides with them, at least at large energy transfers. (c) The FWHM of peak I increases linearly with $q_{\|}$in the limit of low $q_{\|}$, being compatible with 0 at $q_{\|}=0$, and is slightly larger for $q_{\|}$'s along $\overline{\Gamma M}$. For $E_{\text {loss }} \geq 0.5 \mathrm{eV}$, $q_{\|} \geq 0.15 \AA^{-1}$, a change in the slope of the FWHM of peak I vs $q_{\|}$indicates the admixture of further resonances to the loss (see panel B). The generally wider FWHM of peak II may be a further hint that more than one unresolved loss contributes to it. (d) The intensity of 
peak I is nearly the same along both high symmetry directions and decreases exponentially as a function of $q_{\|}$. On the contrary, peak II is more intense along $\overline{\Gamma K}$ (see panel C). (e) As shown in the Supplemental Material [22], these results are confirmed by an additional HREELS investigation. The reason for the different outcome, with respect to the previous study [12], is not evident and could be associated with the nature of the sample, a $\mathrm{Au}(111)$ single crystal, in our case, and a deposited film in Ref. [12].

The central experimental result is thus that the ASP dispersion has a slope half as large as reported previously. This agrees better with general theory, but is still problematic, since it corresponds to an $\alpha$ value smaller than unity, a result again apparently contradicting theory [20].

In order to solve the riddle, time dependent density functional theory calculations of the band structure and of the excitation spectrum of $\mathrm{Au}(111)$ have been performed with a scalar-relativistic code for a 21-layer Au(111) slab. Only few such calculations have been performed previously for films of such thickness, and only for simple metals [11,27-29] and silver [30]. The calculated surface band structure is reported in the Supplemental Material [22] (see Fig. S5). The surface loss function [31,32], given by the imaginary part of the surface response function $g\left(\mathbf{q}_{\|}, \omega\right)$, is related to the dynamical density-response function for interacting electrons $\chi\left(\mathbf{r}, \mathbf{r}^{\prime}, q_{\|}, \omega\right)$ by

$g\left(\mathbf{q}_{\|}, \omega\right)=-\frac{2 \pi}{q_{\|}} \iint d z d z^{\prime} e^{q_{\|}\left(z+z^{\prime}\right)} \chi_{\mathbf{G}=0, \mathbf{G}^{\prime}=0}\left(z, z^{\prime}, \mathbf{q}_{\|}, \omega\right)$.

$\chi$ is obtained from the integral equation $\chi=\chi^{\circ}+\chi v \chi^{\circ}$, where $v$ is a Coulomb potential and $\chi^{\circ}$ the densityresponse function for noninteracting electrons [33]. In the evaluation of $\chi^{\circ}$, all occupied and unoccupied one-particle states up to an energy of $25 \mathrm{eV}$ above the Fermi level have been included and summation over the $2 \mathrm{D}$ Brillouin zone has been performed using a $(144 \times 144) \mathbf{k}_{\|}$grid [27].

The positions of the maxima of the calculated surfaceloss function are reported in Fig. 4 vs $q_{\|}$and compared with experiment. The thick long dashed lines, marked as I, correspond to the (quasilinear) ASP dispersion. In the lowenergy region, we observe an excellent agreement with experiment. As expected, this mode lies very close to the upper limit for the electron-hole intraband excitations within the Shockley SS band (green dotted lines). The surprising observation, however, is that the ASP mode is positioned below this line; i.e., it runs inside the continuum for intraband electron-hole excitations within the Shockley SS band. Referring to Eq. (1), this means that $\alpha<1$.

Such $\alpha$ value can be rationalized by going back to the model of incomplete screening in a two-component electron system. For $\operatorname{Au}(111)$, only the surface state is free electron-like, whereas the bulk bands strongly deviate from this behavior. Our calculations show that around the Fermi level, the bulk density of states has important contributions

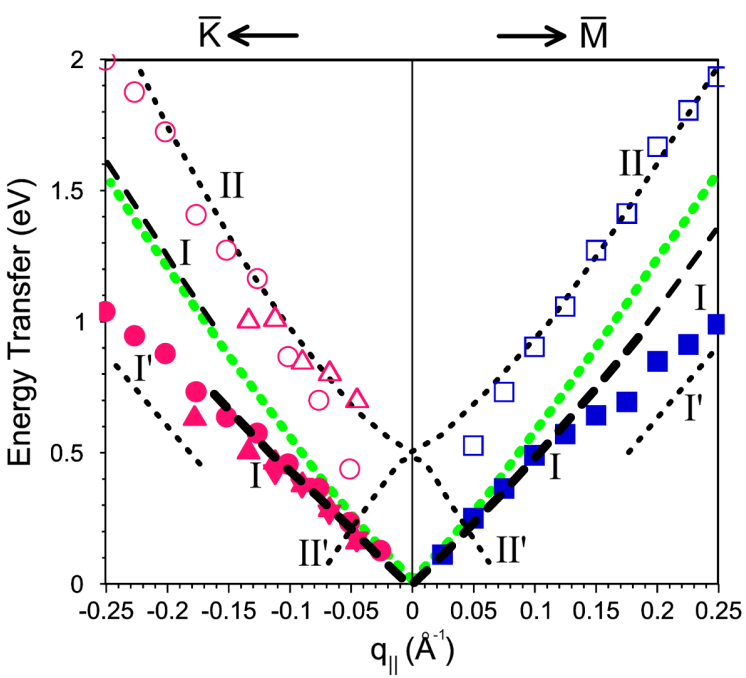

FIG. 4 (color online). Maxima of the calculated surface-loss function $\operatorname{Im} g\left(q_{\|}, \omega\right)$ for the 21 layer $\mathrm{Au}(111)$ slab along $\overline{\Gamma K}$ and $\overline{\Gamma M}$. The thick long-dashed lines (I) correspond to the ASP dispersion. The II (II') peaks highlighted by dotted lines correlate with the upper- (lower-) energy thresholds for interband transitions between the Shockley SS band and the bulk band. In the limit of a semi-infinite crystal, they form a broad feature peaked at their low energy border. The green dotted line represents the upper border of the region of intraband transitions within the Shockley SS band. Symbols correspond to the experimental data of Fig. 3.

from states with velocities significantly smaller than the corresponding velocity of the Shockley SS (see Fig. S6 in the Supplemental Material [22]). This is true over the whole energy range for bulk states with group velocities along the $\overline{\Gamma M}$ direction and in the low-energy range for velocities along $\overline{\Gamma K}$. This means that the situation at $\mathrm{Au}(111)$ is qualitatively different from the previously considered cases $[10,18,34]$. The ASP results now from the incomplete dynamical screening of the majority of slowly moving bulk electrons (those residing in branch B2 of Fig. S6 in the Supplemental Material [22] within the screening length at the surface) by the faster carriers in the Shockley SS band, rather than the contrary. Keeping $v_{\mathrm{F}}^{\mathrm{SS}}$ as a reference in Eq. (1), the coefficient $\alpha<1$.

For momentum transfers along $\overline{\Gamma M}$, the calculated mode I energy disperses almost linearly up to $\approx 1.4 \mathrm{eV}$, with gradually reduced intensity, especially above $\sim 1 \mathrm{eV}$. For $q_{\|}>0.15 \AA^{-1}$, the experimental peak I deviates from theory. Here, however, a further loss I', associated to bulk intraband transitions, starts contributing to the spectrum. Along $\overline{\Gamma K}$, theory predicts the dispersion of the ASP to end, around $0.75 \mathrm{eV}$, where another weaker I peak appears at higher energies dispersing clearly outside of the Shockley SS intraband excitation region. This behavior is indicative of the reestablishment of the "fast bulk-slow surface state" scenario, but this feature, as well as the asymmetry between $\overline{\Gamma M}$ and $\overline{\Gamma K}$ directions, is not resolved 
experimentally, since the data result from the unresolved contributions of modes I and I'. In agreement with this interpretation, a sudden increase of the slope of the experimental FWHM of mode I vs $q_{\|}$is observed beyond the $q_{\|}$ values for which the contribution of mode I' is expected.

In conclusion, the experimentally observed low energy excitation I corresponds to the ASP dispersion, at least up to $0.6 \mathrm{eV}\left(0.15 \AA^{-1}\right)$. At the higher $q_{\|}$values, excitation energies and FWHMs of this loss peak become comparable, so that the distinction between collective and single particle excitations is no longer possible. The second experimentally observed dispersing energy loss (peaks II in Fig. 4) is associated with the upper thresholds for interband transitions between the Shockley SS band and the bulk states (see Fig. S5 in the Supplemental Material [22]). Surprisingly, the ASP turned out to be embedded into the continuum of the surface state intraband electron-hole excitations. Our analysis shows that this puzzling finding is a manifestation of an unusual "slow bulk-fast surface state" scenario taking place at this surface. The $\alpha$ value smaller than unity implies an even larger light confinement capability of Au(111). Since the ASP has a group velocity about 500 times slower than light, the same value can be envisaged for light confinement. We expect these findings to be of relevance for many low-dimensional systems including the recently investigated graphene sheets deposited on transition metal surfaces [16,17,35-37], where extremely slow plasmonic excitations can be present.

We thank K. Pohl for borrowing the $\mathrm{Au}(111)$ sample and Letizia Savio for scientific discussions and we acknowledge the partial support from the University of the Basque Country (Grant No. IT-366-07), the Departamento de Educación del Gobierno Vasco, the Spanish Ministerio de Ciencia e Innovación (Grant No. FIS2010-19609-C02-01), Italian PRIN-MIUR, and Compagnia di San Paolo.

Note added in proof.-After submission of this manuscript a paper [39] has appeared presenting similar $a b$ initio calculation results for the ASP on $\mathrm{Au}(111)$.

[1] W. L. Barnes, A. Dereux, and T.W. Ebbesen, Nature (London) 424, 824 (2003).

[2] S. A. Maier, Plasmonics: Fundamentals and Applications (Springer, New York, 2007).

[3] M. Rocca, Surf. Sci. Rep. 22, 1 (1995).

[4] F. H. L. Koppens, D. E. Chang, and F. J. García de Abajo, Nano Lett. 11, 3370 (2011).

[5] F. Stern, Phys. Rev. Lett. 18, 546 (1967).

[6] J. Chen et al., Nature (London) 487, 77 (2012).

[7] Z. Fei et al., Nature (London) 487, 82 (2012).

[8] H. Yan et al., arXiv:1209.1984.

[9] A. Bill, H. Morawitz, and V.Z. Kresin, Phys. Rev. B 68, 144519 (2003).

[10] V. M. Silkin, A. García-Lekue, J. M. Pitarke, E. V. Chulkov, E. Zaremba, and P. M. Echenique, Europhys. Lett. 66, 260 (2004).
[11] B. Diaconescu et al., Nature (London) 448, 57 (2007).

[12] S. J. Park and R. E. Palmer, Phys. Rev. Lett. 105, 016801 (2010).

[13] K. Pohl, B. Diaconescu, G. Vercelli, L. Vattuone, V. M. Silkin, E. V. Chulkov, P. M. Echenique, and M. Rocca, Europhys. Lett. 90, 57006 (2010).

[14] M. Jahn, M. Müller, M. Endlich, N. Néel, J. Kröger, V. Chis, and B. Hellsing, Phys. Rev. B 86, 085453 (2012)

[15] L. Vattuone, G. Vercelli, M. Smerieri, L. Savio, and M. Rocca, Plasmonics 7, 323 (2012).

[16] T. Langer, D. F. Förster, C. Busse, T. Michely, H. Pfnür, and C. Tegenkamp, New J. Phys. 13, 053006 (2011).

[17] A. Politano, A. R. Marino, V. Formoso, D. Farías, R. Miranda, and G. Chiarello, Phys. Rev. B 84, 033401 (2011).

[18] J. M. Pitarke, V. U. Nazarov, V. M. Silkin, E. V. Chulkov, E. Zaremba, and P. M. Echenique, Phys. Rev. B 70, 205403 (2004).

[19] H. Claus, A. Bussenschutt, and M. Henzler, Rev. Sci. Instrum. 63, 2195 (1992).

[20] D. Pines and P. Nozières, The Theory of Quantum Liquids (Benjamin, New York, 1966), Vol. 1.

[21] T. Ando, A. B. Fowler, and F. Stern, Rev. Mod. Phys. 54, 437 (1982).

[22] See Supplemental Material at http://link.aps.org/ supplemental/10.1103/PhysRevLett.110.127405 for details on the experimental and on the computational methods employed in the present research work.

[23] S. J. Park and R. E. Palmer, Phys. Rev. Lett. 102, 216805 (2009).

[24] http://fityk.nieto.pl/.

[25] R. Paniago, R. Matzdorf, G. Meister, and A. Goldmann, Surf. Sci. 336, 113 (1995).

[26] R. Mazzarello, A. D. Corso, and E. Tosatti, Surf. Sci. 602, 893 (2008).

[27] P. Kowalczyk, W. Kozlowski, W. Olejniczak, and P. K. Datta, Surf. Sci. 600, 1604 (2006).

[28] V. M. Silkin, E. V. Chulkov, and P. M. Echenique, Phys. Rev. Lett. 93, 176801 (2004).

[29] Z. Yuan and S. W. Gao, Comput. Phys. Commun. 180, 466 (2009).

[30] J. Yan, K. S. Thygesen, and K. W. Jacobsen, Phys. Rev. Lett. 106, 146803 (2011).

[31] J. Yan, K. W. Jacobsen, and K. S. Thygesen, Phys. Rev. B 84, 235430 (2011).

[32] B. N. J. Persson and E. Zaremba, Phys. Rev. B 31, 1863 (1985).

[33] A. Liebsch, Electronic Excitations at Metal Surfaces (Plenum Press, New York, 1997).

[34] J.M. Pitarke, V. M. Silkin, E. V. Chulkov, and P. M. Echenique, Rep. Prog. Phys. 70, 1 (2007).

[35] V.M. Silkin, J.M. Pitarke, E. V. Chulkov, and P.M. Echenique, Phys. Rev. B 72, 115435 (2005).

[36] H. Pfnür, T. Langer, J. Baringhaus, and C. Tegenkamp, J. Phys. Condens. Matter 23, 112204 (2011).

[37] A. L. Walter et al., Phys. Rev. B 84, 085410 (2011).

[38] S. Andersson and B. N. J. Persson, Phys. Rev. Lett. 50, 2028 (1983).

[39] J. Yan, K. W. Jacobsen, and K. S. Thygesen, Phys. Rev. B 86, 241404(R) (2012). 